\title{
Synthesis and structure-activity relationship study: Anticancer chalcones derived from 4 '-morpholinoacetophenone
}

\author{
Bedriye Seda KURŞUN-AKTAR, Emine Elçin ORUÇ-EMRE, Ayşegül KARAKÜÇÜK-İYIDOĞAN, Ayse ŞAHİN YAĞLIOĞLU, \\ İbrahim DEMİRTAŞ, Şaban TEKİN
}

\begin{abstract}
The aim of this research was to synthesize chalcone derivatives containing morpholine ring and to investigate their antiproliferative activity. The 3-aryl-1-[4-(morpholin4-yl)phenyl]prop-2-en-1-one derivatives were synthesized and evaluated for their in vitro antiproliferative activities on C6 cells (Rat glioma cell line) and HeLa cells (Human cervix adenocarcinoma), using the BrdU ELISA assay. The calculation of ADME properties of new chalcone derivatives was used by topological polar surface area (TPSA), absorption
\end{abstract}

$(\mathrm{ABS} \%)$ and Lipinski's rules. Most of the compounds exhibited significantly antiproliferative activity on two cell lines. Among the screened compounds, compound 7, 10, 11 and 12 revealed higher antiproliferative activity than cisplatin which was used as reference drug. Compound $\mathbf{1 1}$ on HeLa cell and compound 10 on C6 cell line are the most effective compared with cisplatin.

Keywords: Chalcone; antiproliferative activity; cytotoxic; morpholine
Bedriye Seda Kurşun-Aktar

Yapraklı Vocational School, Çankırı Karatekin University, 18100 Çankırı, Turkey

Emine Elçin Oruç-Emre, Ayşegül Karaküçük-Iyidoğan

Department of Chemistry, Faculty of Arts and Sciences, Gaziantep University, Üniversite Bulvarl, 27310, Gaziantep, Turkey.

Ayse Sahin Yaglıoglu, İbrahim Demirtas,

Department of Chemistry, Faculty of Sciences, Çankırı Karatekin University, Çankırı, Turkey

Şaban Tekin

Department of Biology, Faculty of Arts and Sciences, Gaziosmanpaşa University, Taşlıçiftlik, 60240 Tokat, Turkey.

Corresponding Author:

Emine Elçin Oruç-Emre

e-mail:oruc@gantep.edu.tr

Submitted / Gönderilme: 30.12.2016 Revised / Düzeltme: 20.06.2017

Accepted / Kabul: $\quad 20.06 .2017$

How to cite this article: Kurşun-Aktar BS, Oruç-Emre EE, Karaküçükİyidoğan A, Şahin Yağloğlu A, Demirtaş İ, Tekin Ş. Synthesis and structure-activity relationship study: Anticancer chalcones derived from 4'-morpholinoacetophenone. Marmara Pharm J 2017; 21 (4): 949-960

\section{INTRODUCTION}

Cancer is commonly known as the second major cause of death at a ratio of $20-25 \%$ in developing countries after the cardiovascular disease [1]. The basic principle of the chemotherapy is to inhibit the proliferation and growth of tumor cells without damaging the patient's normal cells and tissues, later to kill tumor cells. But, it is not possible to stop or destroy cancer cells selectively without affecting healthy cells though the physiological and anatomical structure of cancer cells are different from normal cells. Therefore, anticancer drugs which are also cytotoxic agents, cause severe side effects such as hair loss, disorder of gastrointestinal system (vomitting, diarrhea etc.), liver toxication and damage to bone marrow and also drug-resistance to tumor cells occur at long term therapy [2]. Thus, new anticancer agents are urgently needed for cancer therapy.

Flavonoids which are classified as flavonol, flavonone, flavanonol, flavanol, flavone, chalcone etc. are known phytochemical natural productshave different pharmacological activities including anticancer, anti-HIV, antimicrobial, antiinflammatory $[3,4]$. Chalcones are a significant part of plants belonging to the flavonoids family [5]. Two aryl groups are attached by three-carbons with $\alpha, \beta$-unsaturated carbonyl 
system. These compounds have been known to display interesting pharmacological activities such as anticancer, anti-inflammatory, antibacterial, antifungal, antimicrobial, antimalarial and anti-HIV activities [6-13]. Therefore in this research, we synthesized some novel 1,3-diaryl-2-propen-1ones with the hope of discovering more active and selective anticancer agents. The calculation of topological polar surface area (TPSA), absorption (ABS\%) and Lipinski's rule of five the was proved that these compounds 1-12 were drug-likeness compared to cisplatin.

\section{MATERIALS AND METHODS}

\subsection{Chemistry}

All chemicals and solvents were in analytical grade and purchased from Sigma-Aldrich, Merck or Roche. All chemical reactions were monitored with thin layer chromatography (TLC) using Merck silica gel $60 \mathrm{~F}_{254}$ plates. Melting points were determined by EZ-Melt melting point apparatus and were uncorrected. Electronic spectra were recorded in DMF on a PG Instruments T80+ UV-visible Spectrophometer. IR spectra were determined with a Bruker Alpha 4000 FTIR spectrophotometer. Elemental analyses (CHNS) were performed on a VarioMICRO elemental analyzer. ${ }^{1} \mathrm{H},{ }^{13} \mathrm{C}$, DEPT-90, DEPT-135, DEPT, APT, HETCOR, HMBC, NMR spectra were recorded on a Bruker Unity-400 spectrometer. The chemical shift values are given in ppm and following abbreviations were used: $\mathrm{s}=$ singlet, $\mathrm{d}=$ doublet, $\mathrm{dd}=$ doublet to doublet, $\mathrm{t}=$ triplet, $\mathrm{m}=$ multiplet. The coupling constants (J) are given in Hertz. The mass spectra of all compounds were recorded on a Agilent 1260 infinity LC. 6210 HPLCTOF/MS spectrometer in electrospray mode. The mass spectra of compounds 1, $\mathbf{2}$ and $\mathbf{1 2}$ were recorded on a Agilent 1100 MSD spectrometer in order to detect the fragments.

\subsection{General Synthesis Method}

The quantities of substituted benzaldehyde and 4'-morpholino acetophenone were dissolved in methanol, three equivalents of $\mathrm{NaOH}$ was added to the mixture and stirred at the room temperature. The resulting solution was stirred for 2 days and kept in refrigerator overnight. The reaction mixture was extracted with dichloromethane. Organic phase was dried with anhydrous $\mathrm{MgSO}_{4}$. The solvent was evaporated and the crude product recrystallized from $n$-hexane/DCM [14].

2.2.1. (2E)-3-(furan-3-yl)-1-[4-(morpholin-4-yl)phenyl]prop2-en-1-one (1). Green crystal, $69 \%, 180-182^{\circ} \mathrm{C}$. UV/Vis $\lambda_{\max }(\mathrm{nm}): 264,358$. FTIR $v_{\text {max }}\left(\mathrm{cm}^{-1}\right): 1644(\mathrm{C}=\mathrm{O}) ; 1580,1542$, 1515, 1472 (C=C); 1188 (C-O-C morpholine); 1126 (C-N-C morpholine). ${ }^{1} \mathrm{H}-\mathrm{NMR}$ (DMSO-d $\left.{ }_{6}, \delta \mathrm{ppm}\right): 3.32$ (t, $4 \mathrm{H}, \mathrm{Hb}$ morpholine); 3.75 (t, 4H, Ha morpholine); 6.68 (dd, $1 \mathrm{H}$, $J_{1}=2.0 \mathrm{~Hz}, J_{2=} 1.6 \mathrm{~Hz}, \mathrm{Hd} \mathrm{B}$ ring); 7.03 (d, $2 \mathrm{H}, J=8.8 \mathrm{~Hz}, \mathrm{Ha} \mathrm{A}$ ring); 7.06 (d, $1 \mathrm{H}, J=3.2 \mathrm{~Hz}, \mathrm{Hb} \mathrm{B}$ ring); 7.50 (d, $1 \mathrm{H}, J=15.6$ $\mathrm{Hz}, \mathrm{H \alpha}$ proton); 7.56 (d, $2 \mathrm{H}, J=15.2 \mathrm{~Hz}, \mathrm{H} \beta$ proton); 7.89 (d, $1 \mathrm{H}, J=0.8 \mathrm{~Hz}, \mathrm{Hc} B$ ring); 7.99 (d, 2H, $J=9.2 \mathrm{~Hz}, \mathrm{Hb}$ A ring). ${ }^{13} \mathrm{C}-\mathrm{NMR}$ : (100 MHz)(DMSO-d $/ 6$ TMS) $\delta$ ppm: 47.2, 66.3 (- $\left.\mathrm{NCH}_{2} \mathrm{CH}_{2} \mathrm{O}-\right)$; 119.4, 129.4 (-CH=CH-CO-); 113.4, 113.6, 116.4, 127.9, 130.8, 146.2, 151.9, 154.5 (Ar-C); $186.4(\mathrm{C}=\mathrm{O})$. TOF/MS(m/z) $284.1296[\mathrm{M}+\mathrm{H}]^{+}$. MS: $\mathrm{m} / \mathrm{z}=283\left(\mathrm{M}^{+}\right), 255$, $225,197,190,162,132,121,86,77,65,51,39$. Anal. calc. for $\mathrm{C}_{17} \mathrm{H}_{17} \mathrm{NO}_{3}: \mathrm{C}, 72.07 ; \mathrm{H}, 6.05 ; \mathrm{N}, 4.94$. Found: C, 71.84; H, $6.01 ; \mathrm{N}, 5.11 \%$.

2.2.2. (2E)-1-[4-(morpholin-4-yl)phenyl]-3-(thiophen-3-yl) prop-2-en-1-one (2). Yellow crystal, $45 \%, 156-158^{\circ} \mathrm{C}$. UV/Vis $\lambda_{\max }(\mathrm{nm}): 244,227,358$. FTIR $v_{\max }\left(\mathrm{cm}^{-1}\right): 1641(\mathrm{C}=\mathrm{O}) ; 1598$, 1574, 1444, 1422 (C=C); 1188 (C-O-C morpholine); 1120 (C-N-C morpholine). ${ }^{1} \mathrm{H}-\mathrm{NMR}$ (DMSO-d $\left.{ }_{6}, \delta \mathrm{ppm}\right): 3.33$ (t, $4 \mathrm{H}, \mathrm{Hb}$ morpholine), 3.75 (t, 4H, Ha morpholine); 7.04 (d, $2 \mathrm{H}, J=9.2 \mathrm{~Hz}, \mathrm{Ha}$ A ring); 7.19 (dd, $1 \mathrm{H}, J_{1}=3.6 \mathrm{~Hz}, J_{2=} 3.6 \mathrm{~Hz}$, $\mathrm{Hc} \mathrm{B}$ ring); 7.57 (d, $1 \mathrm{H}, J_{1}=15.2 \mathrm{~Hz}$, Ha proton); 7.66 (d, $1 \mathrm{H}$, $J_{1}=3.2 \mathrm{~Hz}, \mathrm{Hb} \mathrm{B}$ ring); 7.76 (d, $1 \mathrm{H}, J=5.2 \mathrm{~Hz}, \mathrm{Hd} \mathrm{B}$ ring); 7.84 (d, $2 \mathrm{H}, J=15.2 \mathrm{~Hz}, \mathrm{H} \beta$ proton); 8.01 (d, 2H, J=9.2 Hz, Hb A ring). ${ }^{13} \mathrm{C}-\mathrm{NMR}:(100 \mathrm{MHz})\left(\mathrm{DMSO}-\mathrm{d}_{6} / \mathrm{TMS}\right) \delta \mathrm{ppm}: 47.2$, 66.3 (- $\left.\mathrm{NCH}_{2} \mathrm{CH}_{2} \mathrm{O}-\right)$; 127.8140 .5 (-CH=CH-CO-); 113.6, 121, 129.1, 130.2, 130.8, 132.5, 135.5, 154.5 (Ar-C); 186.5 $(\mathrm{C}=\mathrm{O}) . \mathrm{TOF} / \mathrm{MS}(\mathrm{m} / \mathrm{z}) 299.0991[\mathrm{M}+\mathrm{H}]^{+} . \mathrm{MS}: \mathrm{m} / \mathrm{z}=299$ $\left(\mathrm{M}^{+}\right), 271,241,213,190,157,137,131,109,86,77,65,51$, 39. Anal. calc. for $\mathrm{C}_{17} \mathrm{H}_{17} \mathrm{NO}_{2} \mathrm{~S}$ : C, 68.20; $\mathrm{H}, 5.72 ; \mathrm{N}, 4.68 ; \mathrm{S}$, 10.09. Found: C, $68.02 ; \mathrm{H}, 5.53 ; \mathrm{N}, 4.46 ; \mathrm{S}, 10.39 \%$.

2.2.3. (2E)-1-[4-(morpholin-4-yl)phenyl]-3-phenylprop-2-en1-one (3). Green crystal, $67 \%, 173-175^{\circ} \mathrm{C}$. UV/Vis $\lambda_{\max }(\mathrm{nm})$ : 227, 264, 304, 354, 357. FTIR $v_{\max }\left(\mathrm{cm}^{-1}\right): 1643(\mathrm{C}=\mathrm{O}) ; 1583$, 1515, 1495, 1445 (C=C); 1220 (C-O-C morpholine); 1118 (C-N-C morpholine). ${ }^{1} \mathrm{H}-\mathrm{NMR}$ (DMSO-d 6 /TMS, $\delta \mathrm{ppm}$ ): 3.33 ( $\mathrm{t}, 4 \mathrm{H}, \mathrm{Hb}$ morpholine); 3.75 ( $\mathrm{t}, 4 \mathrm{H}$, Ha morpholine); 7.04 (d, $2 \mathrm{H}, J=9.2 \mathrm{~Hz}$, Ha A ring); 7.44-7.48 (m, 3H, Hb,c,d $B$ ring); 7.68 (d, $1 \mathrm{H}, J=15.6 \mathrm{~Hz}, \mathrm{Ha}$ proton); 7.88 (dd, $2 \mathrm{H}, J_{1}=$ $J_{2}=2.4 \mathrm{~Hz}, \mathrm{Ha}$,e B ring); 7.94 (d, $1 \mathrm{H}, J=15.6 \mathrm{~Hz}, \mathrm{H} \beta$ proton); 8.09 (d, $2 \mathrm{H}, J=8.8 \mathrm{~Hz}, \mathrm{Hb}$ A ring). ${ }^{13} \mathrm{C}-\mathrm{NMR}:(100 \mathrm{MHz})$ (DMSO-d 6 /TMS) $\delta$ ppm: 47.2, 66.3 (- $\mathrm{NCH}_{2} \mathrm{CH}_{2} \mathrm{O}-$ ); 122.6, 142.7 (-CH=CH-CO-); 113.54, 127.9, 129.14, 129.34, 130.7, 131.04, 135.5, $154.6($ Ar-C); $187(\mathrm{C}=\mathrm{O})$. TOF/MS (m/z) 293.4150 $[\mathrm{M}+\mathrm{H}]^{+}$. Anal. calc. for $\mathrm{C}_{19} \mathrm{H}_{19} \mathrm{NO}_{2}: \mathrm{C}, 77.79 ; \mathrm{H}$, 6.53; N, 4.77. Found: C, 77.50; H, 6.55; N, $4.96 \%$. 
2.2.4. (2E)-3-(4-chlorophenyl)-1-[4-(morpholin-4-yl)phenyl] prop-2-en-1-one (4). Yellow crystal, 69\%, 203-205 C. UV/Vis $\lambda_{\text {max }}(\mathrm{nm}): 230,311,355$. FTIR $v_{\text {max }}\left(\mathrm{cm}^{-1}\right): 1644(\mathrm{C}=\mathrm{O}) ; 1583$, 1487, 1445 (C=C); 1188 (C-O-C morpholine); 1079 (C-N-C morpholine). ${ }^{1} \mathrm{H}-\mathrm{NMR}$ (DMSO- $\left.\mathrm{d}_{6} / \mathrm{TMS}, \delta \mathrm{ppm}\right): 3.36(\mathrm{t}, 4 \mathrm{H}$, $\mathrm{Hb}$ morpholine); 3.89 (t, 4H, Ha morpholine); 6.94 (d, 2H, $J=9.2 \mathrm{~Hz}$, Ha A ring); 7.40 (d, 2H, $J=8.4 \mathrm{~Hz}, \mathrm{Hb}, \mathrm{d}$ B ring); 7.55 (d, 1H, J=15.6 Hz, Ha proton); 7.59 (d, 2H, J=8.4 Hz, Ha,e B ring); 7.76 (d, 1H, J=15,6 Hz, H $\beta$ proton); 8.03 (d, $2 \mathrm{H}, J=9.2 \mathrm{~Hz}, \mathrm{Hb}$ A ring). ${ }^{13} \mathrm{C}-\mathrm{NMR}:(100 \mathrm{MHz})\left(\mathrm{DMSO}_{-} \mathrm{d}_{6} /\right.$ TMS) $\delta$ ppm: 47.4, $66.5\left(-\mathrm{NCH}_{2} \mathrm{CH}_{2} \mathrm{O}-\right) ; 122.4,141.8$ (-CH=CH-CO-); 113.3, 128.6, 129.2, 129.4, 131.2, 133.8, 135.9, 154.3 (Ar-C); 187.8 (C=O). TOF/MS(m/z) 327.1158 $[\mathrm{M}+\mathrm{H}]^{+}$. Anal. calc. for $\mathrm{C}_{19} \mathrm{H}_{18} \mathrm{ClNO}_{2}: \mathrm{C}, 69.62 ; \mathrm{H}, 5.53 ; \mathrm{N}$, 4.27. Found: C, 69.47; H, 5.52; N, $4.57 \%$.

2.2.5. (2E)-3-(2,4-dichlorophenyl)-1-[4-(morpholin-4-yl) phenyl]prop-2-en-1-one (5). Yellow crystal, 42\%. 135-137 ${ }^{\circ} \mathrm{C}$. $\mathrm{UV} / \mathrm{Vis} \lambda_{\max }(\mathrm{nm}): 235,300,355$. FTIR $\nu_{\text {max }}\left(\mathrm{cm}^{-1}\right): 1649(\mathrm{C}=\mathrm{O})$; 1583, 1547, 1464 (C=C); 1186 (C-O-C morpholine); 1120 (C-N-C morpholine). ${ }^{1} \mathrm{H}-\mathrm{NMR}$ (DMSO- $\mathrm{d}_{6} / \mathrm{TMS}, \delta \mathrm{ppm}$ ): 3.35 ( $\mathrm{t}, 4 \mathrm{H}, \mathrm{Hb}$ morpholine), 3.75 ( $\mathrm{t}, 4 \mathrm{H}$, Ha morpholine); 7.04 (d, 2H J=9.2 Hz, Ha A ring); 7.53-7.65 (dd, 1H, $J_{1}=8.8$ $\mathrm{Hz}, J_{2}=2.0 \mathrm{~Hz}, \mathrm{Hb} \mathrm{B}$ ring), 7.74 (d, H, J=2.0 Hz, Hd B ring), 7.90 (d, H, J=15.6 Hz, Ha proton); 8.03 (d, 1H, J=8.8 Hz, Ha B ring); 8.10 (d, 2H, J=9.2 Hz, Hb A ring). 8.30 (d, 1H, J=15.2 $\mathrm{Hz}, \mathrm{H} \beta$ proton). ${ }^{13} \mathrm{C}-\mathrm{NMR}:(100 \mathrm{MHz})\left(\mathrm{DMSO}-\mathrm{d}_{6} / \mathrm{TMS}\right) \delta$ ppm: 47.1, 66.3 (- $\left.\mathrm{NCH}_{2} \mathrm{CH}_{2} \mathrm{O}-\right)$; 126, 136.1 (-CH=CH-CO-); $113.5,127.5,128.3,129.9,130.2,131.3,132.2,135.4,135.6$, 154.7 (Ar-C); $186.5 \quad(\mathrm{C}=\mathrm{O}) . \quad \mathrm{TOF} / \mathrm{MS}(\mathrm{m} / \mathrm{z}) \quad 361.0685$ $[\mathrm{M}+\mathrm{H}]^{+}$. Anal. calc. for $\mathrm{C}_{19} \mathrm{H}_{17} \mathrm{Cl}_{2} \mathrm{NO}_{2}: \mathrm{C}, 63.00 ; \mathrm{H}, 4.73 ; \mathrm{N}$, 3.87. Found: C, 63.17; H, 4.78; N, $4.17 \%$.

2.2.6. (2E)-3-(3,4-dichlorophenyl)-1-[4-(morpholin-4-yl) phenyl]prop-2-en-1-one (6). Yellow crystal, 67\%, 177-179 ${ }^{\circ} \mathrm{C}$. $\mathrm{UV} / \mathrm{Vis} \lambda_{\max }(\mathrm{nm}): 230,235,300,355$. FTIR $v_{\max }\left(\mathrm{cm}^{-1}\right)$ : 1648 (C=O); 1592, 1547, 1516, 1470 (C=C); 1189 (C-O-C morpholine); 1116 (C-N-C morpholine). ${ }^{1} \mathrm{H}-\mathrm{NMR}$ (DMSO- $\left.\mathrm{d}_{6} / \mathrm{TMS}, \delta \mathrm{ppm}\right): 3.36$ (t, 4H, Hb morpholine); 3.89 (t, 4H, Ha morpholine); 6.93 (d, 2H, J=9.2 Hz, Ha A ring); 7.45-7.52 (m, 2H, Hb,e B ring); 7.55 (d, 1H, J=15.6 Hz, Ha proton); 7.69 (d, 1H, J=15.6 Hz, H $\beta$ proton); 7.73 (s, 1H, Ha $\mathrm{B}$ ring); 8.02 (d, 2H, $J=8.8 \mathrm{~Hz}, \mathrm{Hb}$ A ring). ${ }^{13} \mathrm{C}-\mathrm{NMR}$ : (100 $\mathrm{MHz}$ (DMSO-d 6 /TMS) $\delta$ ppm: 47.1, $66.3\left(-\mathrm{NCH}_{2} \mathrm{CH}_{2} \mathrm{O}-\right)$; $126,136.1$ (-CH=CH-CO-); 113.5, 127.5, 128.3, 129.9, 130.2, $131.3,132.2,135.4,135.6,154.7$ (Ar-C); 186.5(C=O). TOF/ $\mathrm{MS}(\mathrm{m} / \mathrm{z}) 361.0645[\mathrm{M}+\mathrm{H}]^{+}$. Anal. calc. for $\mathrm{C}_{19} \mathrm{H}_{17} \mathrm{Cl}_{2} \mathrm{NO}_{2}: \mathrm{C}$, 63.00; H, 4.73; N, 3.97. Found: C, 62.78; H, 4.70; N, $4.12 \%$.
2.2.7. (2E)-3-(4-fluorophenyl)-1-[4-(morpholin-4-yl)phenyl] prop-2-en-1-one (7). Yellow crystal, 70\%, 197-199 C. UV/ Vis $\lambda_{\text {max }}(\mathrm{nm}): 230,306,354,358$. FTIR $v_{\text {max }}\left(\mathrm{cm}^{-1}\right): 1645$ $(\mathrm{C}=\mathrm{O}) ; 1580,1503,1446,1417$ (C=C); 1224 (C-F); 1187 (C-O-C morpholine); 1116 (C-N-C morpholine). ${ }^{1} \mathrm{H}-\mathrm{NMR}$ (DMSO-d ${ }_{6} / \mathrm{TMS}, \delta \mathrm{ppm}$ ): 3.33 (t, 4H, Hb morpholine), 3.75 (t, 4H, Ha morpholine); 7.04 (d, 2H, $J=9.2 \mathrm{~Hz}$, Ha A ring); 7.30 (d, 2H, Ha,e B ring); 7.70 (d, 1H, J=15.6 Hz, Ha proton); 7.91 (d, $1 \mathrm{H}, J=15.2 \mathrm{~Hz}, \mathrm{H} \beta$ proton); 7.94-7.98 (m, $2 \mathrm{H}, \mathrm{Hb}, \mathrm{d}$ B ring); 8,09 (d, 2H, J=9.2 Hz, Hb A ring). ${ }^{13} \mathrm{C}-\mathrm{NMR}$ : (100 $\mathrm{MHz}$ ) (DMSO-d $/$ TMS) $\delta$ ppm: 47.2, 66.3 (- $\mathrm{NCH}_{2} \mathrm{CH}_{2} \mathrm{O}-$ ); 122.6, 141.5 (-CH=CH-CO-); 116.2, 116.4, 127.9, 131, 131.4, 132.2, 154.6, 162.4 (Ar-C); $186.9(\mathrm{C}=\mathrm{O}) . \mathrm{TOF} / \mathrm{MS}(\mathrm{m} / \mathrm{z})$ $311.1459[\mathrm{M}+\mathrm{H}]^{+}$. Anal. calc. for $\left(\mathrm{C}_{19} \mathrm{H}_{18} \mathrm{FNO}_{2}\right): \mathrm{C}, 73.29 ; \mathrm{H}$, 5.83; N, 4.50; Found: C, 73.37; H, 5.85; N, $4.81 \%$.

2.2.8. (2E)-3-[4-(methylsulfanyl)phenyl]-1-[4-(morpholin4-yl)phenyl]prop-2-en-1-one (8). Yellow crystal, 71\%, 145$147^{\circ} \mathrm{C}$. UV/Vis $\lambda_{\max }(\mathrm{nm}): 227,250,358$. FTIR $v_{\max }\left(\mathrm{cm}^{-1}\right)$ : 1646 (C=O); 1580, 1548, 1489, 1443 (C=C); 1219 (C-O-C morpholine); 1115 (C-N-C morpholine). ${ }^{1} \mathrm{H}-\mathrm{NMR}\left(\mathrm{CDCl}_{3}\right.$, $\delta \mathrm{ppm}): 2.54\left(\mathrm{~s}, 3 \mathrm{H},-\mathrm{SCH}_{3}\right) ; 3.36$ (t, 4H, Hb morpholine); 3.89 (t, 4H, Ha morpholine); 6.94 (d, 2H, J=9.2 Hz, Ha A ring); 7.27 (d, 2H, J=8.0 Hz, Hb,d B ring); 7.55-7.59 (m, 3H, Ha,e B ring, Ha proton); 7.78 (d, $1 \mathrm{H}, J=15.6 \mathrm{~Hz}, \mathrm{H} \beta$ ); 8.03 (d, $2 \mathrm{H}, J=8.8 \mathrm{~Hz}$, Hb A ring). ${ }^{13} \mathrm{C}-\mathrm{NMR}:(100 \mathrm{MHz})\left(\mathrm{CDCl}_{3}\right.$ ,TMS) $\delta$ ppm: 14.69 (-SCH$)$; 47.21, $66.33\left(-\mathrm{NCH}_{2} \mathrm{CH}_{2} \mathrm{O}-\right)$; $186.9(\mathrm{C}=\mathrm{O}) ; 141.8,122.4$ (-CO-CH=CH-); 154.3, 135.9, 133.8, 131.2, 129.4, 129.2, 128.6, 113.3 (Ar-C). TOF/MS(m/z) $340.1344[\mathrm{M}+\mathrm{H}]^{+}$. Anal. calc. for $\mathrm{C}_{20} \mathrm{H}_{21} \mathrm{NO}_{2} \mathrm{~S}: \mathrm{C}, 70,77 ; \mathrm{H}$, 6.24; N, 4.13; S, 9.45. Found: C, 70,24; H, 6.03; N, 5.09; S, $9.88 \%$.

2.2.9. (2E)-3-(4-methoxyphenyl)-1-[4-(morpholin-4-yl) phenyl]prop-2-en-1-one (9). Yellow crystal, $79 \%, 138-140^{\circ} \mathrm{C}$. $\mathrm{UV} / \mathrm{Vis} \lambda_{\max }(\mathrm{nm}): 227,354,368,372$. FTIR $v_{\max }\left(\mathrm{cm}^{-1}\right): 1643$ $(\mathrm{C}=\mathrm{O}) ; 1598,1564,1509,1445$ (C=C); 1224 (C-F); 1193 (C-O-C morpholine); 1117 (C-N-C morpholine). ${ }^{1} \mathrm{H}-\mathrm{NMR}$ (DMSO- $\mathrm{d}_{6} / \mathrm{TMS}, \delta \mathrm{ppm}$ ): 3.34 (t, 4H, Hb morpholine); 3,87 $\left(\mathrm{s}, 3 \mathrm{H},-\mathrm{OCH}_{3}\right) ; 3.88$ (t, 4H, Ha morpholine ring); 6,93 (d, $2 \mathrm{H}, J=8.8 \mathrm{~Hz}$, Ha A ring); 6,95 (d, 2H, $J=8.0 \mathrm{~Hz} \mathrm{Hb}, \mathrm{d}$ B ring); 7.47 (d, 1H, J=15.6 Hz, Ha proton); 7,62 (d, 2H, J=8.4 Hz, Ha,e B ring); 7.80 (d, 1H, J=15,6 Hz, H $\beta$ proton ); 8.03 (d, $2 \mathrm{H}, J=8.8 \mathrm{~Hz}, \mathrm{Hb}$ A ring). ${ }^{13} \mathrm{C}-\mathrm{NMR}$ : (100 MHz) (DMSO- $\mathrm{d}_{6} /$ TMS) $\delta$ ppm: 42.8, 50.6, 61.9 (Aliphatic C); 114.8, 138.4 (-CH=CH-CO-); 108.7, 109.6, 124.4, 123.2, 125.2, 125.8, 149.3, 156.6 (Ar-C); 188.2 (C=O). TOF/MS(m/z) 325.1666 $[\mathrm{M}+\mathrm{H}]^{+}$. Anal. calc. for $\mathrm{C}_{20} \mathrm{H}_{21} \mathrm{NO}_{3}: \mathrm{C}, 74.28 ; \mathrm{H}, 6.55 ; \mathrm{N}, 4.33$. Found: C, 74.00; H, 6.54; N, $4.47 \%$. 
2.2.10. (2E)-1-[4-(morpholin-4-yl)phenyl]-3-(4-nitrophenyl) prop-2-en-1-one (10). Orange crystal, $53 \%, 143-145^{\circ} \mathrm{C}$. UV/ Vis $\lambda_{\text {max }}(\mathrm{nm}): 227,312,388$. FTIR $v_{\max }\left(\mathrm{cm}^{-1}\right): 1654(\mathrm{C}=\mathrm{O})$; 1594, 1448, 1420 (C=C); 1190 (C-O-C morpholine); 1108 (C-N-C morpholine). ${ }^{1} \mathrm{H}-\mathrm{NMR}$ (DMSO-d $\mathrm{d}_{6} / \mathrm{TMS}, \delta \mathrm{ppm}$ ): 3.34 (t, $4 \mathrm{H}, \mathrm{Hb}$ morpholine); 3.75 (t, 4H, Ha morpholine); 7.05 (d, 2H, J=9.2 Hz, Ha A ring); 7.75 (d, $1 \mathrm{H}, J=15.2 \mathrm{~Hz}$, Ha proton); 8.10 (d, 2H, J=8.8 Hz, Ha,e B ring); 8.12 (d, 2H, $J=8.8 \mathrm{~Hz}, \mathrm{Hb}$ A ring); 8.15 (d, $1 \mathrm{H}, J=14.4 \mathrm{~Hz}, \mathrm{H} \beta$ proton); 8.28 (d, $2 \mathrm{H}, J=8.8 \mathrm{~Hz}, \mathrm{Hb}, \mathrm{d}$ B ring). ${ }^{13} \mathrm{C}-\mathrm{NMR}:(100 \mathrm{MHz})$ (DMSO-d 6 /TMS) $\delta$ ppm: 47.1, $66.3\left(-\mathrm{NCH}_{2} \mathrm{CH}_{2} \mathrm{O}-\right) ; 126.8$, 142.0 (-CH=CH-CO-); 113.5, 124.3, 127.5, 130, 131.3, 139.8, 148.3, 154.7 (Ar-C); $186.6(\mathrm{C}=\mathrm{O})$. The HMBC spectrum displayed correlations of $\mathrm{H}-4 / \mathrm{C}-3$; H-4/C-5; H-5/C-6; H-8/C-7; H-8/C-9; H-8/C-10; H-9/C-7; H-9/C-8; H-9/C-10; $\mathrm{H}-12 / \mathrm{C}-10 ; \mathrm{H}-12 / \mathrm{C}-13$. In DEPT 90 spectrum, only CH's signal was dedected and it was positive. Therefore $\mathrm{CH}$ positive signals of $\mathbf{1 0}$ were detected at 113.46, 124.34, 126.80, $130.07,131.27,139.88 \mathrm{ppm}$. The negative signals in DEPT 135 were indicated that $\mathrm{CH}_{2}$ group of morpholine were at 47.15 and $66.3 \mathrm{ppm}$. TOF/MS (m/z) $338.1327[\mathrm{M}+\mathrm{H}]^{+}$. Anal. calc. for $\mathrm{C}_{19} \mathrm{H}_{18} \mathrm{~N}_{2} \mathrm{O}_{4}: \mathrm{C}, 67.44 ; \mathrm{H}, 5.36 ; \mathrm{N}, 8.28$. Found: C, 67.40; H, 5.31; N, $8.24 \%$.

2.2.11. (2E)-1-[4-(morpholin-4-yl)phenyl]-3-[4(trifluoromethyl)phenyl]prop-2-en-1-one (11). Yellow crystal, $71 \%, 130-132^{\circ} \mathrm{C}$. UV/Vis $\lambda_{\max }(\mathrm{nm}): 227,292,358$. FTIR $v_{\max }\left(\mathrm{cm}^{-1}\right): 1651(\mathrm{C}=\mathrm{O}) ; 1585,1517,1449,1416(\mathrm{C}=\mathrm{C}) ; 1159$ (C-O-C morpholine); 1104 (C-N-C morpholine). ${ }^{1} \mathrm{H}-\mathrm{NMR}$

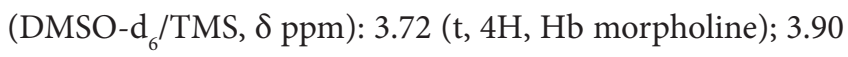
(t, $4 \mathrm{H}, \mathrm{Ha}$ morpholine); 6.95 (d, $2 \mathrm{H}, J=8.8 \mathrm{~Hz}, \mathrm{Ha}$ A ring); 7.63-7.69 (m, 3H, Ha,e B ring, Ha proton); 7.76 (d, 2H, J=8.0 $\mathrm{Hz}, \mathrm{Hb}, \mathrm{d} \mathrm{B}$ ring); 7.81 (d, $1 \mathrm{H}, J=15.6 \mathrm{~Hz}, \mathrm{H} \beta$ proton); 8.04 (d, $2 \mathrm{H}, J=9.2 \mathrm{~Hz}, \mathrm{Hb}$ A ring). ${ }^{13} \mathrm{C}-\mathrm{NMR}$ : (100 MHz)(DMSO-d 6 / TMS) $\delta$ ppm: 47.4, 66.5 (- $\left.\mathrm{NCH}_{2} \mathrm{CH}_{2} \mathrm{O}-\right) ; 123.5,140.4$ (-CH=CH-CO-); 113.4，127.5，128.3，129.6，130.7，130.8, 133.5, 135.4, $154.4($ Ar-C); $187.4(\mathrm{C}=\mathrm{O})$. TOF/MS (m/z) $362.1358[\mathrm{M}+\mathrm{H}]^{+}$. Anal. calc. for $\mathrm{C}_{20} \mathrm{H}_{18} \mathrm{~F}_{3} \mathrm{NO}_{2}: \mathrm{C}, 66.48 ; \mathrm{H}$, 5.02; N, 3.88. Found: C, 66.71; H, 5.05; N, $4.18 \%$.

2.2.12. (2E)-1-[4-(morpholin-4-yl)phenyl]-3-[4(trifluoromethoxy)phenyl]prop-2-en-1-one (12). Yellow crystal, $75 \%, 119-121^{\circ} \mathrm{C}$. UV/Vis $\lambda_{\max }(\mathrm{nm}): 227,297,358$. FTIR $v_{\text {max }}\left(\mathrm{cm}^{-1}\right): 1647(\mathrm{C}=\mathrm{O}) ; 1602,1581,1504,1448(\mathrm{C}=\mathrm{C})$; 1157 (C-O-C morpholine); 1119 (C-N-C morpholine). ${ }^{1} \mathrm{H}-\mathrm{NMR}$ (DMSO-d $/ 6$ TMS, $\delta \mathrm{ppm}$ ): 3.33 (t, $4 \mathrm{H}, \quad \mathrm{Hb}$ morpholine); 3.74 (t, 4H, Ha morpholine); 7.04 (d, 2H, J=9.2 $\mathrm{Hz}$, Ha A ring); 7.44 (d, 2H, Hd,c B ring); 7.69 (d, 1H, J=15.6 $\mathrm{Hz}, \mathrm{Ha}$ proton); 7.97 (d, $1 \mathrm{H}, J=15.6 \mathrm{~Hz}, \mathrm{H} \beta$ proton); 8.02 (d, $2 \mathrm{H}, J=8.8 \mathrm{~Hz}, \mathrm{Hb}$ A ring); 8.09 (d, $2 \mathrm{H}, J=8.8 \mathrm{~Hz}, \mathrm{Ha}, \mathrm{e} B$ ring). ${ }^{13} \mathrm{C}-\mathrm{NMR}:(100 \mathrm{MHz})\left(\mathrm{DMSO}-\mathrm{d}_{6} / \mathrm{TMS}\right) \delta \mathrm{ppm:}$ 47.2, 66.3 (- $\left.\mathrm{NCH}_{2} \mathrm{CH}_{2} \mathrm{O}-\right)$; $123.8,140.9$ (-CH=CH-CO-); 113.5, 119.2, 121.7, 127.8, 131.02, 131.1, 134.8, 149.7, 154.6 (Ar-C); $186.9(\mathrm{C}=\mathrm{O}) . \mathrm{TOF} / \mathrm{MS}(\mathrm{m} / \mathrm{z}) 377.1464[\mathrm{M}+\mathrm{H}]^{+}$. MS: $\mathrm{m} / \mathrm{z}=$ 377( $\left.\mathrm{M}^{+}\right), 349,292,291,215,190,187,132,105,103,102,86$, 77, 65, 51. Anal. calc. for $\mathrm{C}_{20} \mathrm{H}_{18} \mathrm{~F}_{3} \mathrm{NO}_{3}: \mathrm{C}, 63.66 ; \mathrm{H}, 4.81 ; \mathrm{N}$, 3.71. Found: C, $64.16 ; \mathrm{H}, 4.84 ; \mathrm{N}, 3.96 \%$.

\subsection{Cell Culture Studies}

Cisplatin was used as positive control. Stock solution of compounds were prepared in DMSO and diluted with Dulbecco's modified eagle medium (DMEM). DMSO final concentration is below $1 \%$ in all tests. C6 cells (Rat glioma cell 1 ine) and HeLa cells (Human cervix adenocarcinoma) were kindly provided by Prof.Dr. Nazlı Arda and Associate Prof. Ali Karagoz (Department of Molecular Biology, Istanbul University, Turkey). The cancer cell lines were grown in Dulbecco's modified eagle medium (DMEM) supplemented with $10 \%$ fetal bovine serum (FBS), $2 \%$ penicilin streptomycin. The medium was changed twice a week.

\subsubsection{Antiproliferative Activity Assay}

Antiproliferative effects of the chalcones were investigated on HeLa and C6 cell lines using BrdU ELISA assay kit $(15,16)$. Cultured cells were grown in 96-well plates (COSTAR, Corning, USA) at a density of $3 \times 10^{4}$ cells well $^{-1}$. In each experimental set, cells were plated in triplicate and replicated twice. The cell lines were determined in each compounds with eight concentrations (at 5, 10, 20, 30, 40, 50, 75 and $100 \mu \mathrm{M}$ ) as well as positive controls DMSO and cisplatin, for $24 \mathrm{~h}$ at $37^{\circ} \mathrm{C}$ in a humidified atmosphere of $5 \% \mathrm{CO}_{2}$. Cells were then incubated for overnight before applying the $\mathrm{BrdU}$ Cell Proliferation ELISA assay reagent (Roche, Germany) according to manufacturer's procedure. The amount of cell proliferation was assessed by determining the A450 nm by using a microplate reader (Ryto, China). Results were reported as percentage of the inhibition of cell proliferation, where the optical density measured from vehicle-treated cells was considered to be $100 \%$ of proliferation. All assays were repeated at least twice using HeLa and C6 cell lines. Percentage of inhibition of cell proliferation was calculated as follows: $\left[1-\left(\mathrm{A}_{\text {samples }} / \mathrm{A}_{\text {control }}\right)\right] \times 100$.

\subsection{Cell cytotoxicity}

LDH assay was carried out using LDH cytotoxicity detection kit (Roche Diagnostics GmbH, Mannheim, Germany) according to protocol in the user's manual. The highest dose was used 
as the concentration of $100 \mu$ land determined cytotoxicity (\%) on HeLa cell line. Samples and 5-FU (5-fluorouracil) were incubated with $100 \mu \mathrm{l}$ of HeLa cell suspension having $5 \times 10^{3}$ cell $^{*} \mathrm{ml}^{-1}$ in 96 well plate at $37{ }^{\circ} \mathrm{C}$ for overnight in $5 \% \mathrm{CO}_{2}$ atmosphere. All the control and tested substances were tested in triplicates and twice and mean \pm SEM of the absorbance value were taken to calculate cytotoxicity.

Cytotoxicity $\%=($ Triplicate absorbance - low control $) /($ High control - low control) x 100

\subsection{Statistical analysis}

The results of investigation in vitro are means $\pm \mathrm{SD}$ of nine measurement. Differences between groups were tested with ANOVA. $p$ values of $<0.01$ were considered as significant.

The half maximal inhibitory concentration $\left(\mathrm{IC}_{50}\right)$ is a measure of the effectiveness of a compound in inhibiting biological function. In this paper, $\mathrm{IC}_{50}$ and $\mathrm{IC}_{75}$ values were determined using ED50 plus v1.0.

\section{RESULTS AND DISCUSSION}

\subsection{Synthesis of compounds}

3-aryl-1-[4-(morpholin-4-yl)phenyl]prop-2-en-1-ones were synthesized by Claisen-Schmidt condensation between 4'-morpholineacetophenone and various benzaldehydes in the presence of $\mathrm{NaOH}$ in methanol (Scheme 1). 1-12 were purified by hexane-DCM mixture. The yields were in the range of $45-79 \%$. 3, 4, 7 and 9 were synthesized by Gopalakrishnan et al [17]. Chalcones which were colored compound observed three absorption bands at 227-235,
250-311 and 355-372 $\mathrm{nm}$. The band at 355-372 $\mathrm{nm}$ indicated the presence of cinnamyl group. The absorption band at 227$235 \mathrm{~nm}$ due to benzoyl groups, were evaluated according to the exchange of chromophore groups. The IR spectrum showed the characteristic bands in the region of 1661-1641 $\mathrm{cm}^{-1}$ indication a carbonyl band as previously reported [7]. Aromatic and aliphatic $=\mathrm{C}-\mathrm{H}$ streching bands were observed at $3180-2982 \mathrm{~cm}^{-1}$ and $2970-2835 \mathrm{~cm}^{-1}$ respectively. The aromatic $\mathrm{C}-\mathrm{Cl}$ and $\mathrm{C}-\mathrm{F}$ streching band were at 1105-1090 $\mathrm{cm}^{-1}$ and $1235-1120 \mathrm{~cm}^{-1}$ respectively [18].

In the ${ }^{1} \mathrm{H}$ NMR spectrum, protons at $\alpha, \beta$ unsaturated carbonyl system of 1-12 resonated doublet at 7.47-7.90 ppm and the other doublet at 7.56-8.09 ppm which confirmed the presence of chalcone moiety. The coupling constants of the vincylic system $(J=15.2-15.6 \mathrm{~Hz})$ confirmed the trans configuration of the chalcones. The ${ }^{1} \mathrm{H}$ NMR spectra of 8 and 9 carrying $-\mathrm{SCH}_{3}$ and $-\mathrm{OCH}_{3}$ groups showed a $\mathrm{CH}_{3}$ signals at 2.54 and $3.87 \mathrm{ppm}$, respectively. ${ }^{13} \mathrm{C}$ NMR spectra of 1-12 displayed signals in the range 42.8 and $66.3 \mathrm{ppm}$ which represent the methylene $\left(\mathrm{CH}_{2}\right)$ group of morpholine of the chalcone moiety. The ${ }^{13} \mathrm{C}$ NMR spectra indicated that the carbonyl signal was at 186.4-188.2 ppm. It is known that the signals of $\mathrm{CH}$ and $\mathrm{CH}_{3}$ are positive while the $\mathrm{CH}_{2}$ signal is negative in the DEPT135 spectrum. In DEPT 90 spectrum, only CH's signal is detected and positive. Therefore, CH's positive signals of $\mathbf{1 2}$ were detected at 113.3, 121.69, 123.89, $140.9,131.1,131.03 \mathrm{ppm}$. The negative signals in DEPT 135 were indicated that the $\mathrm{CH}_{2}$ group of morpholine were at 47.15 and $66.3 \mathrm{ppm}$ (Figure 1a). The HMBC spectrum of 12 displayed correlations of $\mathrm{H}-5 / \mathrm{C}-7$; H-5/C-6; H-4/C-3; H-4/C-8; H-9/C-7; H-9/C-10; H-8/C-7; H-8/C-10; H-8/C-5; H-11/C-10; H-12/C-14 (Figure $1 \mathrm{~b}$ and 1c).

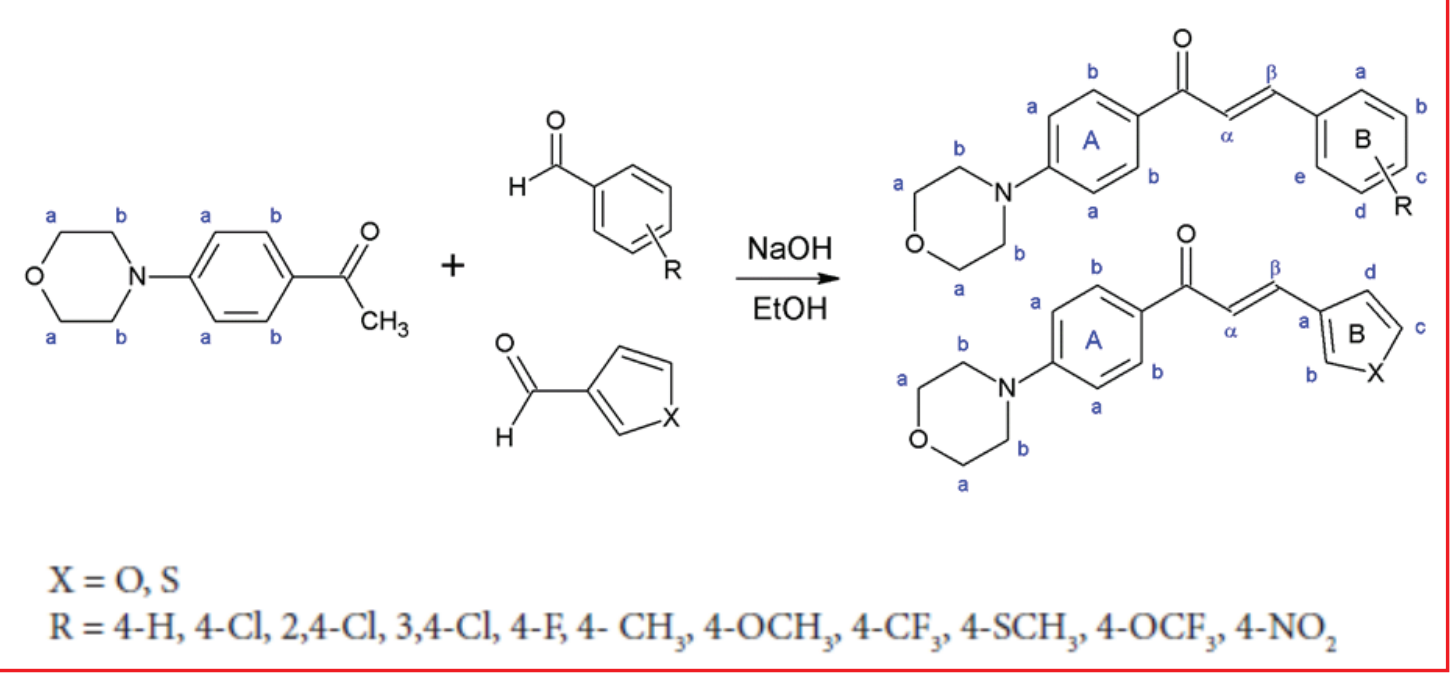

Scheme 1. General synthetic pathway 

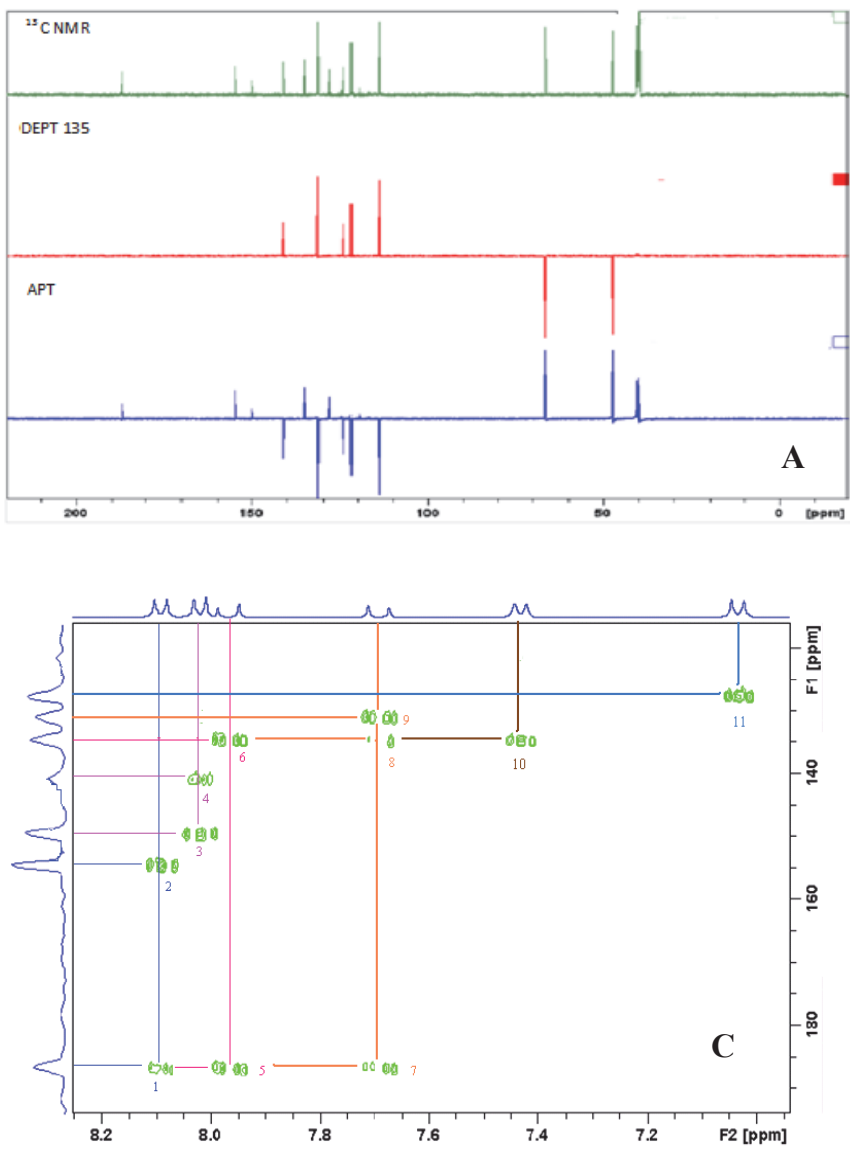

The mass fragmentation of compound containing thiophene and furan rings were according to literature $[18,19]$. The cleavage between carbonyl group and 4-morpholinophenyl ring gave the fragment at $\mathrm{m} / \mathrm{z} 163$ and also the fragmentation of the single bond between carbonyl and ethylene group produced an ion $\mathrm{m} / \mathrm{z} 190$ (Scheme 2). The loss of $-\mathrm{OCF}_{3}$ $(\mathrm{m} / \mathrm{z} 85)$ became the base peak of spectrum of compound 12. The mass spectrum of morpholine showed that the ring was cleaving as a result of the loss of carbonyl group as given in literature [20,21]. And another important fragment was the cleavage of the morpholine ring $(\mathrm{m} / \mathrm{z} 86)$ from the structure (compound 1, 2 and 12).

\subsection{Antiproliferative activity results}

The 3-aryl-1-[4-(morpholin-4-yl)phenyl]prop-2-en-1-ones 1-12 were evaluated in vitro for their antiproliferative activity on two cancer cell lines, HeLa and C6 cells, using the BrdU ELISA assay (Table 1). The activities of compounds 3, 4, 7, 8, $9,10,11,12$ that have substituent at the four position of the phenyl ring were compared with cisplatin and 5-FU (Figure

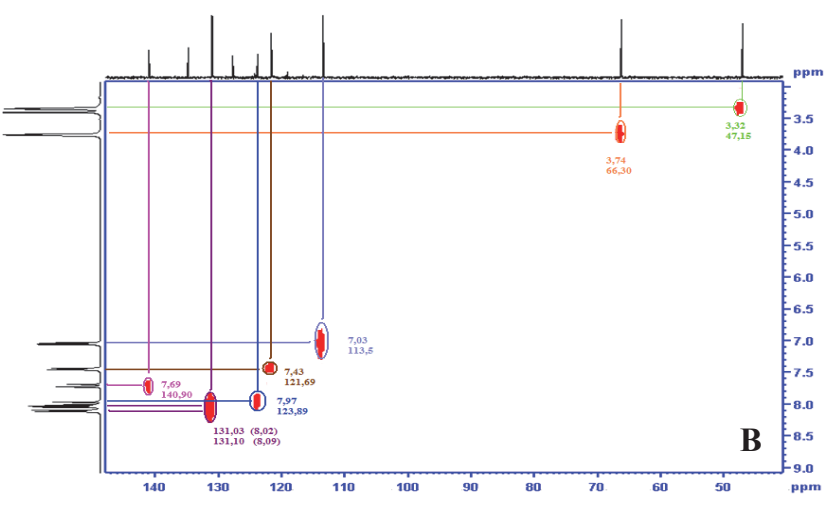

Figure 1. The ${ }^{13} \mathrm{C}$ NMR, DEPT 135 and APT spectrum (A) ; HETCOR spectrum (B); HMBC spectrum $(\mathrm{C})$ of compound $\mathbf{1 2}$ 2a). According to Figure 2a, the antiproliferative activities of the substituents on HeLa cell line showed the following order $($ at $5 \mu \mathrm{M}): 11>12>7>9>5$-FU $>10>8>$ cisplatin $>4>3$, i.e., $\mathrm{CF}_{3}>\mathrm{OCF}_{3}>\mathrm{F}>\mathrm{OCH}_{3}>5-\mathrm{FU}>\mathrm{NO}_{2}>\mathrm{SCH}_{3}>$ cisplatin $>$ $\mathrm{Cl}>\mathrm{H}$ to compared with cisplatin and 5-FU. This results showed that $\mathrm{F}$ atom was important for activity. However, the activities of compounds 1 and 2 (at $5 \mu \mathrm{M}$ ) which were the bioisomer of phenyl ring the antiproliferative effects of thiophene ring were the higher than furan ring on HeLa cell line (Figure 2b). According to Figure 2c, compound $\mathbf{5}$ which has 2,4-dichlorophenyl ring have higher activity than compound 4 which has 4-chloro phenyl ring and compound 3 which has phenyl ring.

All compounds showed higher activities than cisplatin. The antiproliferative effects of substituents were secondly investigated on C6 cell line. The activities of 3, 4, 7, 8, 9, 10, 11, 12 which had para subtituent at phenyl ring were compared with between themselves. According to Figure 3a, the antiproliferative activities of the substituents aganist C6 cell line showed the following order (at $5 \mu \mathrm{M}): 5-\mathrm{FU}>10>12>$ $11>$ cisplatin $>3>8>7>4>9$, i.e., $5-\mathrm{FU}>p-\mathrm{NO}_{2}>p-\mathrm{OCF}_{3}>$ 


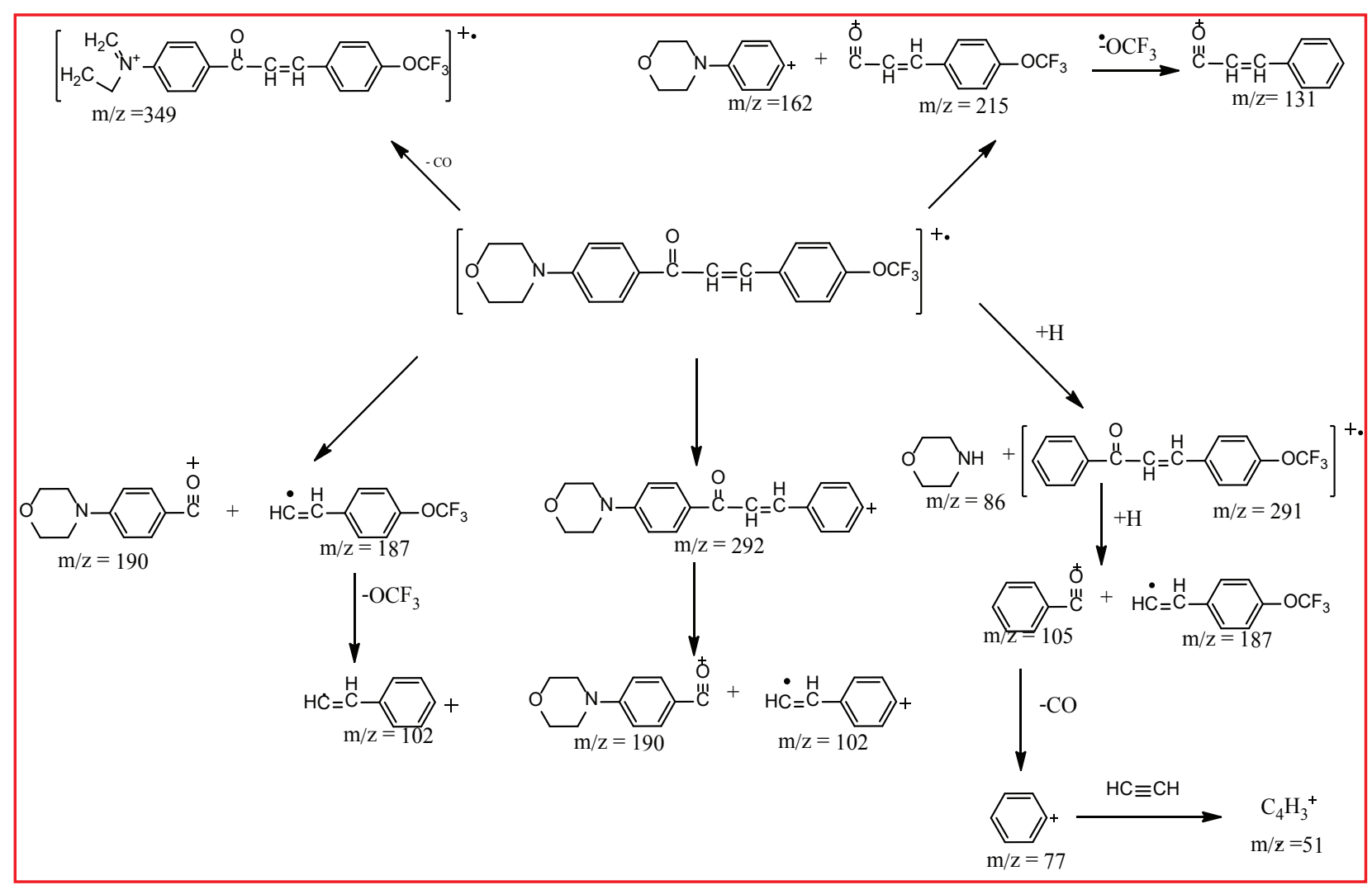

Scheme 2. The mass fragmentation of compound $\mathbf{1 2}$ which was selected as a model compound

Table 1. $\mathrm{IC}_{50}$ and $\mathrm{IC}_{75}$ values of samples and cisplatina)

\begin{tabular}{|c|c|c|c|c|}
\hline \multirow[t]{2}{*}{ Compounds } & \multicolumn{2}{|l|}{$\mathrm{HeLa}$} & \multicolumn{2}{|l|}{$\mathrm{C} 6$} \\
\hline & $I C_{50}[\mu \mathrm{M}]$ & $I C_{75}[\mu \mathrm{M}]$ & $I C_{50}[\mu \mathrm{M}]$ & $I C_{75}[\mu \mathrm{M}]$ \\
\hline 1 & $\left.-{ }^{-b}\right)$ & 30.39 & - & 36.55 \\
\hline 2 & - & 19.09 & 2.77 & 45.80 \\
\hline 3 & 68.27 & 79.16 & 7.36 & 45.32 \\
\hline 4 & 24.31 & 49.42 & 10.31 & 47.89 \\
\hline 5 & - & 7.77 & - & 13.86 \\
\hline 7 & - & 12.66 & - & 39.50 \\
\hline 8 & - & 32.65 & 14.50 & 54.26 \\
\hline 9 & - & 14.95 & 18.93 & 50.43 \\
\hline 10 & - & 29.26 & - & 42.37 \\
\hline 11 & - & - & - & 49.48 \\
\hline 12 & - & - & - & 34.42 \\
\hline Cisplatin & 43.89 & 68.28 & 19.54 & 54.54 \\
\hline 5-FU & - & 5.77 & - & 26.08 \\
\hline
\end{tabular}


against HeLa cell

$\square 3 \square 4 \square 7 \square 8 \square 9 \square 10 \square 11 \square 12 \square$ Cisplatin $\square 5-F U$

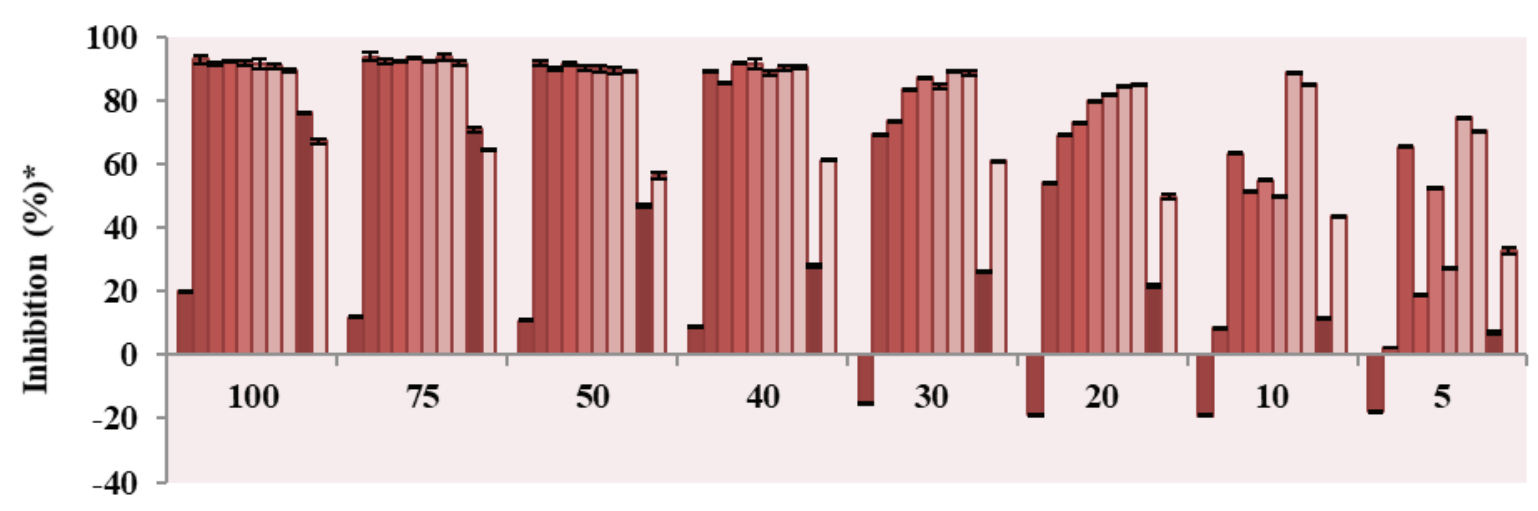

Concentration $(\mu \mathrm{M})$

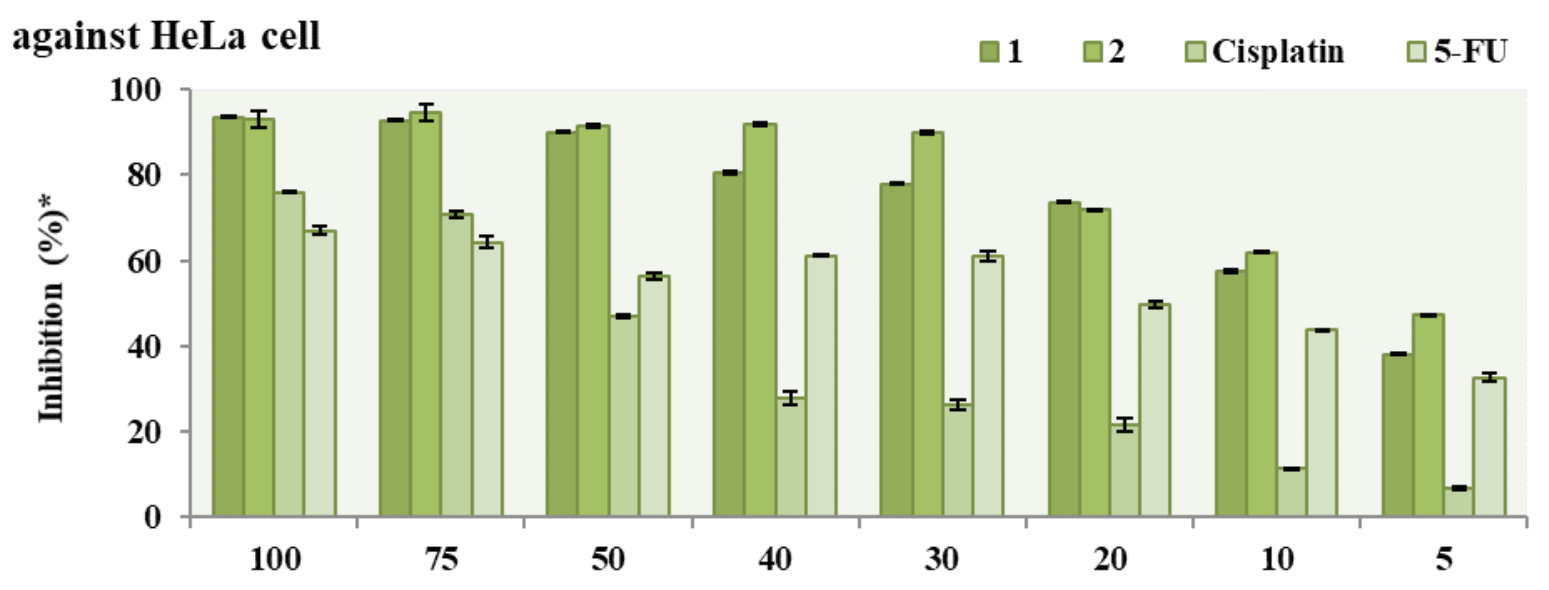

Concentration $(\mu \mathrm{M})$

against HeLa cell

$\square 3 \square 4 \square 5 \square$ Cisplatin $\square 5-F U$

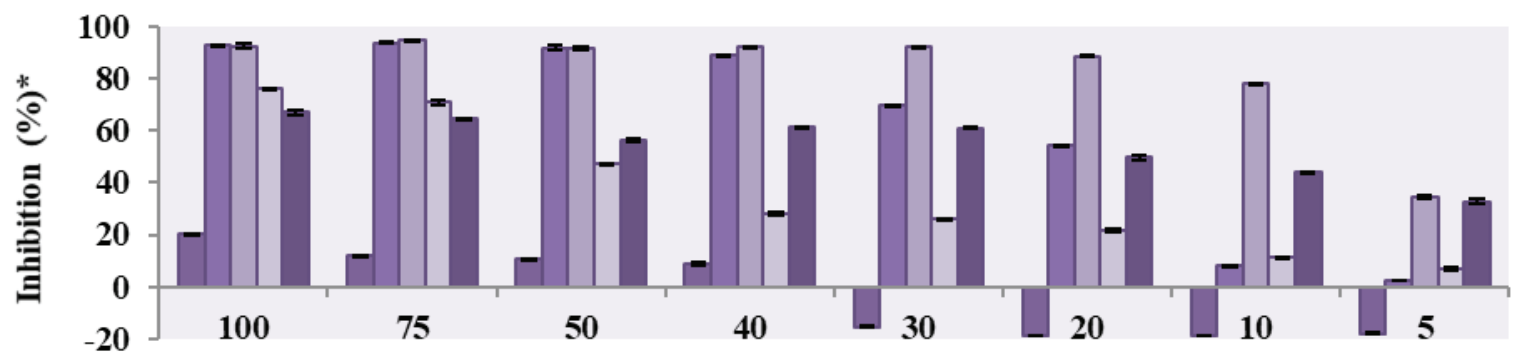

Concentration $(\mu \mathrm{M})$

Figure 2. Antiproliferative activity of samples, cisplatin and 5-FU on HeLa cell line. 

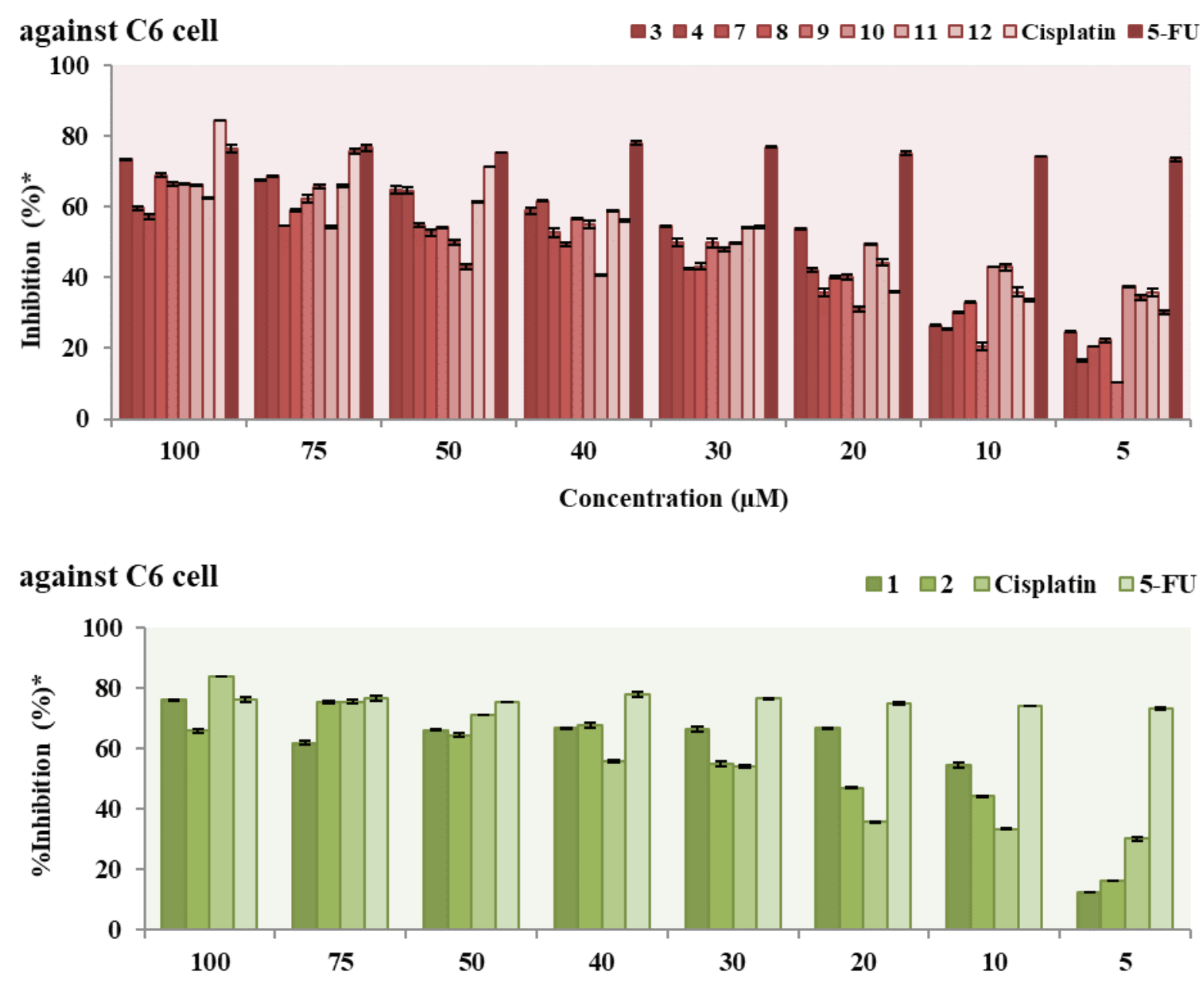

Concentration $(\mu \mathrm{M})$

against $\mathrm{C} 6$ cell

$\square 3 \square 4 \square 5 \square$ Cisplatin $\square 5-F U$

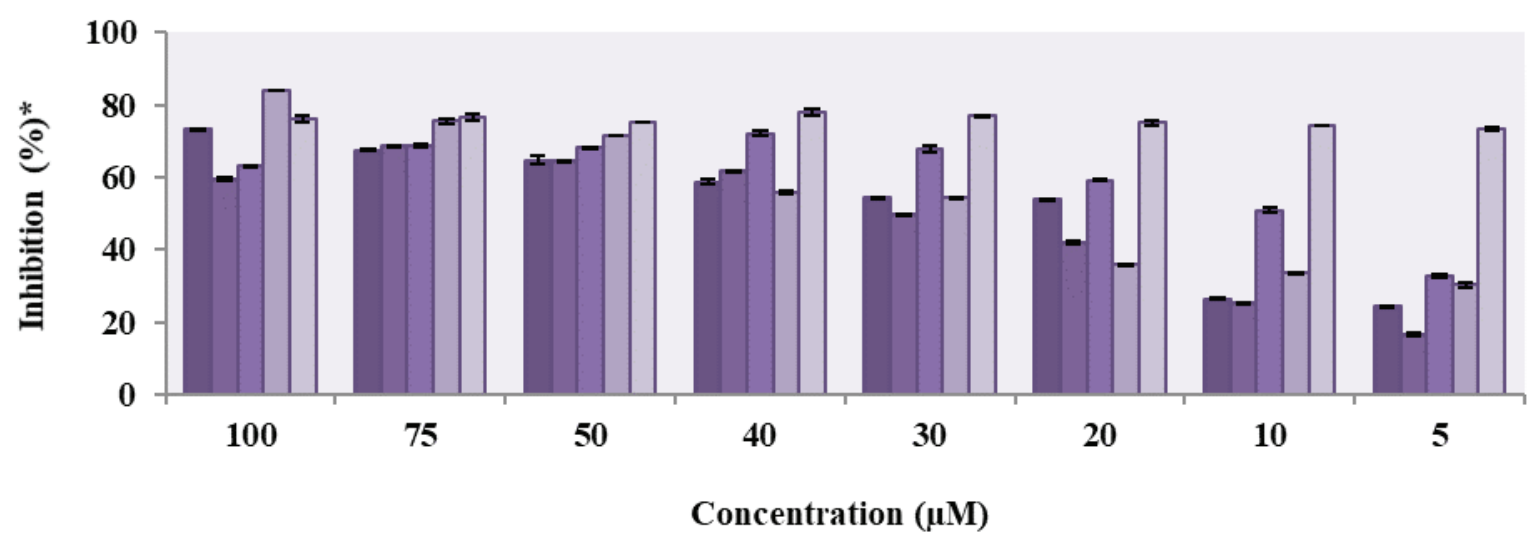

Figure 3. Antiproliferative activity of samples, cisplatin and 5-FU on C6 cell line. 
$p-\mathrm{CF}_{3}>$ cisplatin $>\mathrm{H}>p-\mathrm{SCH}_{3}>p-\mathrm{F}>\mathrm{p}-\mathrm{Cl}>p-\mathrm{OCH}_{3}$. The substitution of $\mathrm{NO}_{2}$ in phenyl ring $\mathbf{1 0}$ increased the inhibitor activity as compared to the standard cisplatin. Sato et al. reported that some compounds carrying nitro group could not show carcinogenicity and mutagenicity [22]. But in our research, our nitro compound $\mathbf{1 0}$ had potent antiproliferative activity. Organofluorine compounds are gained much more importance in the cancer therapy [23]. In our study, 7, 11 and 12 having flour atom, showed the highest activity on HeLa cell line.

However, according to Figure $3 \mathrm{~b}$, the antiproliferative effects of thiophene ring were the higher than furan ring on C6 cell line (at $5 \mu \mathrm{M}$ ). Compound 5 which had 2,4-dichlorophenyl ring showed higher activities than 4 which has 4-chlorophenyl ring and compound $\mathbf{3}$ which has phenyl ring (Figure 3c).

\subsection{Cytotoxic effects results}

At $100 \mu \mathrm{g} / \mathrm{mL}$ concentration that was the highest dose at antiprolifertaive activity test were determined cytotoxicity (\%) on HeLa cell line. 5-FU were used as positive control. Test results were given (Table 2). According to results, cytotoxicity (\%) values of 2 were shown as quite low than 5-FU. In addition to, cytotoxicity (\%) values of $\mathbf{1 0}$ was the same with cytotoxicity (\%) values 5-FU. Low to high compared with 5 -FU toxicity values were as follows respectively: compound 2>4 5-FU $>6>7>5 \sim 9>8>12>11$.
Table 2. Cytotoxicity (\% ) values of samples on HeLa cell.

\begin{tabular}{lc}
\hline Compound & ${\text { Cytotoxicity }(\%)^{*}}^{*}$ \\
\hline 1 & 18 \\
2 & 1 \\
5 & 18 \\
6 & 6 \\
7 & 17 \\
8 & 27 \\
9 & 18 \\
10 & 4 \\
11 & 38 \\
12 & 28 \\
5 -FU & 4 \\
\hline
\end{tabular}

*each substance was tested twice in triplicates aganist cell lines. Data show avarage of 2 individual experiments $(\mathrm{p}<0.01$ for cytotoxicity (\% ) values of all samples)

\subsection{Druglikeness properties}

The number of hydrogen bond acceptors ( $\mathrm{n}-\mathrm{ON})$ and donors $(\mathrm{n}-\mathrm{OHNH})$ are within the Lipinski's rules, $\mathrm{n}-\mathrm{ON}<10$ and $\mathrm{n}-\mathrm{OHNH}<5$. The calculated $\log \mathrm{P}$ must be smaller than 5 . In our study the $\log \mathrm{P}$ values of all synthesized compounds were smaller than 5 except compound $6(\log \mathrm{P}: 5.043)$. The molecular weight of chalcone derivatives are at the range of 283.327 $\mathrm{Da}$ and 377.362 Da. These derivatives could be a new potential anticancer agents according to calculated data. The calculation of absorption (\% ABS) according to Zhao et al. (24), TPSA of compound 1-12 and Lipinski's rule of five were given (Table 3).

Table 3 . Drug-likeness properties of the synthesized chalcone derivatives *)

\begin{tabular}{cccccccccc}
\hline Compound & logP & TPSA & \% ABS & MW & nON & nOHNH & Solubility & Drug likeness & Drug-score \\
\hline $\mathbf{1}$ & 3.168 & 29.543 & 98.807 & 299.395 & 3 & 0 & -3.84 & 2.73 & 0.47 \\
$\mathbf{2}$ & 2.526 & 42.683 & 94.274 & 283.327 & 4 & 0 & -3.6 & 2.72 & 0.5 \\
$\mathbf{3}$ & 3.76 & 29.543 & 98.807 & 293.366 & 3 & 0 & -3.94 & -0.33 & 0.34 \\
$\mathbf{4}$ & 4.437 & 29.543 & 98.807 & 327.811 & 3 & 0 & -4.68 & 3.43 & 0.4 \\
$\mathbf{5}$ & 4.864 & 29.543 & 98.807 & 362.256 & 3 & 0 & -5.42 & 3.79 & 0.33 \\
$\mathbf{6}$ & 5.043 & 29.543 & 98.807 & 362.256 & 3 & 0 & -5.42 & 3.09 & 0.32 \\
$\mathbf{7}$ & 3.923 & 29.543 & 98.807 & 311.356 & 3 & 0 & -4.26 & 1.75 & 0.34 \\
$\mathbf{8}$ & 4.193 & 29.543 & 98.807 & 339.46 & 3 & 0 & -4.79 & 2.92 & 0.39 \\
$\mathbf{9}$ & 3.816 & 38.777 & 95.622 & 323.392 & 4 & 0 & -3.96 & 2.89 & 0.46 \\
$\mathbf{1 0}$ & 3.718 & 75.367 & 82.998 & 338.363 & 6 & 0 & -4.45 & 0.46 & 0.17 \\
$\mathbf{1 1}$ & 4.655 & 29.543 & 98.807 & 361.363 & 3 & 0 & -4.72 & -4.69 & 0.2 \\
$\mathbf{1 2}$ & 4.729 & 38.777 & 95.622 & 377.362 & 4 & 0 & -4.97 & -7.97 & 0.18 \\
\hline
\end{tabular}

*) These parameters were determined with Molinspiration Calculation software and Preadmet software 


\section{CONCLUSIONS}

We reported the synthesis and evaluation of a series of antiproliferative activity carrying 2-propen-1-one group. The results of the activity studies showed that the most of the synthesized chalcone derivatives had antiproliferative activities on two cell lines, especially HeLa compared with cisplatin. (2E)-1-[4-(morpholin-4-yl)phenyl]-3-(4nitrophenyl)prop-2-en-1-one 10 for C6 cell line and (2E)1-[4-(morpholin-4-yl)phenyl]-3-[4-(trifluoromethyl) phenyl]prop-2-en-1-one $\mathbf{1 1}$ for HeLa cell line were the most effective compounds than cisplatin with comparison of $\mathrm{IC}_{50}$.

All these examples showed antiproliferative activity results were quite remarkable, and \% cytotoxicity in combination with low values of the results increased the importance of processing.

\section{ACKNOWLEDGEMENTS}

This work was supported by Gaziantep University Scientic Research Projects Governing Unit (BAPYB) (Grant no: FEF.11.06, Gaziantep, Turkey). This study forms part of the theses of B.S.Kurşun, master's student in Organic Chemistry.

\section{Appendix A. Supplementary Material}

Supplementary material related to this article can be accessed at http://dx.doi.org/10.12991/mpj.2017.18.

\section{REFERENCES}

1. Jaracz S, Chen J, Kuznetsova LV, Ojima I. Recent advances in tumor-targeting anticancer drug conjugates. Bioorg Med Chem 2005; 13: 5043-54.

2. Sashidhara KV, Kumar A, Kumar M, Sarkar J, Sinha S. Synthesis and in vitro evaluation of novel coumarin -chalcone hybrids as potential Anticancer agents. Bioorg Med Chem 2010; 24: 7205-11.

3. Hatzade K, Taile V, Gaidhane P, Ingle V. Synthesis, structural determination, and biological activity of new 7-hydroxy-3pyrazolyl-4H-chromen- 4-ones and their o-b-D-glucosides. Turk J Chem 2010; 34: 241-54.

4. Campos-Buzzi F, Padaratz P, Meira AV, Corrêa R, Nunes RJ, Cechinel-Filho V. 4'-Acetamidochalcone derivatives as potential antinociceptive agents. Molecules 2007; 12: 896-906.

5. Modzelewska A, Pettit C, Achanta G, Davidson NE, Huang P, Khan SR. Anticancer activities of novel chalcone and bischalcone derivatives. Bioorg Med Chem 2006; 14: 3491-5.
6. Zhang L, Wang X, Wang J, Grinberg N, Krishnamurthy DK, Senanayake $\mathrm{CH}$. An improved method of amide synthesis using acyl chlorides. Tetrahedron Lett 2009; 50: 2964-6.

7. Bandgar BP, Gawande SS, Bodade RG, Gawande NM, Khobragade CN. Synthesis and biological evaluation of a novel series of pyrazole chalcones as anti-inflammatory, antioxidant and antimicrobial agents. Bioorg Med Chem 2009;17: 8168-73.

8. Tiwari V, Ali P, Meshram J. Microwave assisted synthesis of 3-(2-chloroguiolin-3-yl)-1-substituted phenyl prop-2-en-1ones using $\mathrm{K}_{2} \mathrm{CO}_{3}$ as a mild, cheap and inexpensive catalyst. Int J Chem Tech Res 2010; 2: 1031-5.

9. Boeck P, Falcao CAB, Leal PC, Yunes RA, Filho VC, TorresSantos EC, Rossi-Bergmann B. Synthesis of chalcone analogues with increased antileishmanial activity. Bioorg Med Chem 2006; 14: 1538-45.

10. Ceylan M, Gurdere MB, Karaman I, Gezegen H. Synthesis and screening antimicrobial activities of novel 1,3-diaryl-3(phenylthio)propan-1-one derivatives. Med Chem Res 2011; 20: 109-15.

11. Mishra N, Arora P, Kumar B, Mishra LC, Bhattacharya A, Awasthi SK, Bhasin VK. Synthesis of novel substituted 1,3-diaryl propenone derivatives and their antimalarial activity in vitro. Eur J Med Chem 2008; 43: 1530-5.

12. Wheeler $\mathrm{OH}$, Gore $\mathrm{PH}$, Santiago $\mathrm{M}$, Baez R. Ultraviolet Absorption of Substituted Phenyl and Polycyclic aryl Chalcones. Canadian J Chem 1964; 42: 2580-3.

13. Rizvi SUF, Siddiqui HL, Johns M, Detorio M, Schinazi RF. Anti-HIV-1 and cytotoxicity studies of piperidyl-thienyl chalcones and their 2-pyrazoline derivatives. Med Chem Res 2012; $21: 3741-9$.

14. a) Kursun BS, Oruç-Emre EE, Karaküçük-İyidoğan A, Şahin A, Tekin Ş, Demirtaş İ. Antiproliferative activity of the substituted morpholine derivatives chalcone. Turkish Patent Institute, TPE 2011/05601, 2011. b) Kursun BS, Oruç-Emre EE, Karaküçük-İyidoğan A, Şahin A, Tekin Ş, Demirtaş İ. Antiproliferative activity of the substituted morpholine derivatives chalcone. Turkish Patent Institute, TPE 2013/04957, 2013.

15. Demirtas I, Sahin A. Bioactive volatile content of the stem and root of Centaurea carduiformis DC. subsp. carduiformis var. carduiformis. E-J Chem 2012; 2013.

16. Demirtas I, Sahin A, Ayhan B, Tekin S, Telci I. antiproliferative effects of the methanolic extracts of Sideritis libanotica Labill. subsp. linearis. Rec Nat Prod 2009; 3: 104-9.

17. Gopalakrishnan M, Thanusu J, Kanagarajan V, Govindaraju R. Synthesis, antibacterial, and antifungal activities of biolabile (E)-1-(4-morpholinophenyl)-3-aryl-prop-2-en-1ones. Med Chem Res 2009; 18: 341-50.

18. Silverstein RM, Bassler GC, Merrill TC. Spectrometric Identification of Organic Compounds. John Wiley \& Sons,Inc, Singapore. 1991.

19. Palibroda N. Electron ionisation mass spectra of some substituted chalcones. Studia Universitatis Babes-Bolyai, Physica, L 2005; 4b: 34-38.

20. Okwu DE, Ukanwa N. Isolation and characterization of flavonoids chalcones and anthocynidines from bridelia ferruginea benth. Der Chemica Sinica 2010; 1: 21-8. 
21. Azev YA, Dülcks T, Gabel D. Cyanuric and thiocyanuric esters as carriers of boron-containing fragments and their fragmentation in mass spectrometry. Tetrahedron Lett 2003; 344: 8689-91.

22. Sato T, Ashizawa N, Iwanaga T, Nakamura H, Matsumoto K, Inoue T, Nagata O. Design, synthesis, and pharmacological and pharmacokinetic evaluation of 3-phenyl-5-pyridyl1,2,4-triazole derivatives as xanthine oxidoreductase inhibitors. Bioorg Med Chem Lett 2009; 19:184-7.

23. Luzina EL, Popov AV. Synthesis and anticancer activity of $\quad N$-bis(trifluoromethyl)alkyl- $N$ '-thiazolyl and $N$-bis(trifluoromethyl)alkyl- $N$ '-benzothiazolyl ureas. Eur J Med Chem 2009; 44: 4944-53.

24. Zhao YH, Abraham MH, Le J, Hersey A, Luscombe CN, Beck G, Sherborne B, Cooper I. Rate-limited steps of human oral absorption and QSAR studies. Pharm Res 2012;19: 1446-57. 